\title{
Impact of methoxyacetic acid on mouse Leydig cell gene expression
}

\author{
Gargi Bagchi, Yijing Zhang and David J Waxman*
}

\begin{abstract}
Background: Methoxyacetic acid (MAA) is the active metabolite of the widely used industrial chemical ethylene glycol monomethyl ether, which is associated with various developmental and reproductive toxicities, including neural toxicity, blood and immune disorders, limb degeneration and testicular toxicity. Testicular toxicity is caused by degeneration of germ cells in association with changes in gene expression in both germ cells and Sertoli cells of the testis. This study investigates the impact of MAA on gene expression in testicular Leydig cells, which play a critical role in germ cell survival and male reproductive function.
\end{abstract}

Methods: Cultured mouse TM3 Leydig cells were treated with MAA for 3, 8, and $24 \mathrm{~h}$ and changes in gene expression were monitored by genome-wide transcriptional profiling.

Results: A total of 3,912 MAA-responsive genes were identified. Ingenuity Pathway analysis identified reproductive system disease, inflammatory disease and connective tissue disorder as the top biological functions affected by MAA. The MAA-responsive genes were classified into 1,366 early responders, 1,387 mid-responders, and 1,138 late responders, based on the time required for MAA to elicit a response. Analysis of enriched functional clusters for each subgroup identified 106 MAA early response genes involved in transcription regulation, including 32 genes associated with developmental processes. 60 DNA-binding proteins responded to MAA rapidly but transiently, and may contribute to the downstream effects of MAA seen for many mid and late response genes. Genes within the phosphatidylinositol/phospholipase C/calcium signaling pathway, whose activity is required for potentiation of nuclear receptor signaling by MAA, were also enriched in the set of early MAA response genes. In contrast, many of the genes responding to MAA at later time points encode membrane proteins that contribute to cell adhesion and membrane signaling.

Conclusions: These findings on the progressive changes in gene expression induced by MAA in a cultured Leydig cell model may help elucidate signaling pathways that lead to the testicular pathophysiological responses induced by MAA exposure and may identify useful biomarkers of MAA toxicity.

\section{Background}

Methoxyacetic acid (MAA) is the primary active metabolite of the industrial chemical ethylene glycol monomethyl ether, a component of paints, inks, varnishes and anti-icing additive in jet fuels [1]. MAA exposure is associated with various developmental and reproductive toxicities in both rodents and humans, including decreased sperm production reflecting increased apoptosis of primary spermatocytes [2] and is accompanied by gene expression changes in germ cells (reviewed in [3]). How-

* Correspondence: djw@bu.edu

1 Division of Cell and Molecular Biology, Department of Biology, Boston University, Boston, MA 02215, USA

Full list of author information is available at the end of the article ever, the precise testicular cell target(s) of MAA that lead to the observed increase in germ cell apoptosis are uncertain. The survival and proper functioning of germ cells requires cooperation of several testicular cell types, including Sertoli cells, which nurture the developing germ cells through spermatogenesis [4], and Leydig cells, the major site of testosterone production in males [5]. MAA-induced changes in gene expression in Sertoli and Leydig cells could therefore have a significant impact on germ cell behavior and overall reproductive function. While MAA-induced changes in Sertoli cell gene expression have been described [6], the impact of MAA on Leydig cell gene expression has not been investigated. 
Environmental chemicals that interfere with normal Leydig cell gene expression have the potential to impact germ cell function. Leydig cell lines have been useful for investigating the testicular actions of environmental chemicals, including effects on gene expression [7], and in the case of MAA, changes in gene expression have been investigated using the cultured TM3 Leydig cell model, which is derived from the testis of the immature $\mathrm{Balb} / \mathrm{c}$ mouse [8]. In particular, MAA was found to alter the expression of TM3 cell genes involved in testosterone biosynthesis (Cyp17a1) and androgen binding (Shbg) [9]. Presently, we use this same Leydig cell model to characterize the sequential changes in gene expression that occur following MAA exposure. Genome-wide transcriptional profiling was carried out to elucidate the global impact of MAA treatment at each of three time points (3, 8 and 24 h) in an effort to identify both early (primary) and late (secondary) MAA response genes. Cells were treated with MAA at $5 \mathrm{mM}$, corresponding to the plasma concentration associated with ethylene glycol monomethyl ether-induced germ cell toxicity in mice [10], and at $1 \mathrm{mM}$, to identify genes affected at a concentration of MAA similar to that seen in exposed humans [11]. A total of 3,912 genes responsive to $5 \mathrm{mM}$ MAA treatment were identified, 1,629 of which were also responsive at $1 \mathrm{mM}$ MAA. The early MAA-responsive genes include 106 genes involved in transcriptional regulation, whereas many of the genes responding to MAA at later time points encode membrane proteins that contribute to cell adhesion and membrane signaling. These MAA-induced perturbations of cellular and biological functions may help elucidate the signaling pathways perturbed by this environmental toxicant and explain its mechanism of action at the gene level.

\section{Methods}

\section{Chemicals and reagents}

MAA and horse serum were purchased from Sigma Chemical Co. (St. Louis, MO). DMEM-F12 culture medium, fetal bovine serum (FBS) and TRIzol reagent were purchased from Invitrogen Corp. (Carlsbad, CA).

\section{MAA treatment of TM3 cells and RNA analysis}

Mouse TM3 Leydig cells (American Type Culture Collection, Manassas, VA) were grown in DMEM-F12 medium containing 5\% horse serum and 2.5\% FBS. Cells were grown to $\sim 60 \%$ confluence and treated with culture medium alone, or with culture medium containing $1 \mathrm{mM}$ or 5 mM MAA for either 3,8 or $24 \mathrm{~h}$. Total RNA was then isolated using TRIzol reagent, followed by incubation with RQ1 RNAse-free DNAse for $1 \mathrm{~h}$ at $37^{\circ} \mathrm{C}$ and then heat inactivation at $75^{\circ} \mathrm{C}$ for $5 \mathrm{~min}$. A total of 6 cultures of TM3 cells were independently treated with MAA under each of the 6 treatment conditions specified above (i.e., 1
mM or 5 mM MAA for either 3,8 or $24 \mathrm{~h}$ ), and the corresponding 36 RNA samples were validated by RNA integrity analysis (Agilent Bioanalyzer). Each RNA sample was also validated in terms of the MAA response by qPCR analysis using SYBR Green I-based chemistry [12] and primers specific for 3 genes known to respond to MAA (Cyp17a1, Shbg, and Igfbp3; [9]) to verify consistency of the MAA responses. Dissociation curves were examined after each qPCR run to ensure amplification of a single, specific product at the correct melting temperature. The 6 RNA samples for each treatment condition were then used to prepare two independent pools ( $n=3$ RNA samples each) for microarray analysis with dye swaps, as described below. Microarray results were validated for 6 genes, three of which were induced at both $1 \mathrm{mM}$ and 5 mM MAA, and three of which were repressed at $5 \mathrm{mM}$ MAA. Data are presented as the expression of the gene of interest relative to an $18 \mathrm{~S}$ RNA internal control in the MAA-treated sample compared with the untreated control, as determined using the comparative $\mathrm{Ct}$ method [13], and are based on duplicate RNA samples for each of three independent experiments for each condition of MAA treatment, with all six samples assayed in triplicate (mean \pm SE for $n=3$ ).

\section{Microarray analysis}

The Agilent Whole Genome Mouse Microarray platform (catalog G4122F; Agilent Technology) was used to characterize MAA-induced changes in TM3 cell gene expression. This array contains 41,174 mouse probes (features), each consisting of a 60-mer oligonucleotide. Accession numbers were obtained for 39,355 out of the 41,174 probes, of which 33,011 were assigned gene names. An additional 3,570 probes were assigned gene names using the microarray probe annotation tool AILUN [14] to map microarray probes to Entrez genes [15]. Each probe corresponding to a distinct mouse transcript is referred to as representing a separate gene/gene product. RNA samples pooled from 3 separate TM3 cell cultures were analyzed in a total of six separate competitive hybridization experiments, corresponding to the above specified combinations of exposure time $(3 \mathrm{~h}, 8 \mathrm{~h}$, and $24 \mathrm{~h})$ and MAA concentration ( $1 \mathrm{mM}, 5 \mathrm{mM})$. Sample labeling, hybridization to microarrays, scanning and calculation of normalized expression ratios were carried out at the Wayne State University Institute of Environmental Health Sciences microarray facility using Alexa 555 and Alexa 647 aminoallyl-aRNA samples as described [16]. Dye swap experiments were carried out using a separate pool of three MAA-treated TM3 cultures. To carry out the dye swaps, Alexa 555-labeled RNA from one of the MAA-treatment conditions was mixed with Alexa 647-labeled RNA from the untreated control at the same time point, and vice versa. TIFF images of each scanned slide were analyzed 
using Agilent's feature extraction software with linear and LOWESS normalization followed by combination of dyeswap samples and calculation of weighted ratios and $p$ values based on the Rosetta error model using Rosetta Resolver (version 5.1, Rosetta Biosoftware, Seattle, WA) [17]. Features flagged in either channel were excluded from analysis. The final set of expression ratios and $p$-values is available as GEO series GSE-20625.

\section{Statistical analysis}

In those cases where two or more probes mapped to the same gene accession and gave the same pattern of expression across all microarrays (reflecting probe redundancy in the array platform), a single representative probe was retained in the final data set. Of the 41,174 probes included on the array, 33,940 non-redundant probes were identified for the 5 mM MAA treatment data set. The statistical significance of differential expression of each corresponding transcript was determined by application of a filter $(p<0.005)$ to the Rosetta-generated $p$-values. Next, an absolute $\log _{2}$ ratio filter of $>2$ SD above the mean value was combined with the above $p$-value filter to determine the number of gene transcripts that were differentially regulated at any of the three time points. In total, 3,912 probes met the combined thresholds for differential expression in at least one of the three $5 \mathrm{mM}$ MAA time points. Of the 33,940 non-redundant probes, 5,031 met the $>2$ SD differential expression filter for at least one of the three microarray comparisons. The number of probes expected to meet the combined threshold $(p<0.005$ and $>2$ SD fold-change expression) by chance is $0.005 \times 5,031$, or 25 probes. The actual number of probes meeting the combined threshold was 3,912, corresponding to an apparent FDR of $25 / 3,912$, or $0.64 \%$. Commonly used multiple testing correction methods such as Bonferroni or Holm step-down were not applied as these eliminate a large number of true positives and introduce an inappropriate over correction.

A system of binary and decimal flags was used to cluster the differentially regulated genes into subgroups based on expression ratios [18]. Briefly, all genes that met both the fold change and the statistical significance threshold criteria (average ratio $>2$, and $p<0.005$ ) for one or more of the three $5 \mathrm{mM}$ MAA treatment conditions were assigned a binary flag value of 1,2, 4 respectively. The sum of these binary flag values defines the whole number portion of the flag assigned to each gene and indicates which of the three microarrays met the specified threshold criteria in our analysis. In addition, decimal values of $0.1,0.01,0.001$ or $0.2,0.02,0.002$, were respectively assigned to each of the three microarrays to indicate the direction of regulation of the genes in the array (decimal flags with values of 1 indicate up-regula- tion, whereas those with a value of 2 indicate down-regulation). Thus, for each gene, the Total Flag Sum (TFS), comprising the binary sum plus the decimal values, indicates which of the three arrays met the threshold criteria for inclusion and the direction of regulation. A similar flag system was used to identify common response genes at $1 \mathrm{mM}$ and $5 \mathrm{mM}$, with by extending the TFS to six binary flags $(1,2,4,8,16$ and 32$)$ and six decimal values $(0.1,0.01,0.001,0.0001,0.00001,0.000001$ or $0.2,0.02$, $0.002,0.0002,0.00002,0.000002)$, except that in that case the average ratio threshold was set at $>2 \mathrm{SD}$, corresponding to a fold change of $>2$-fold for the $5 \mathrm{mM}$ MAA data set and $>1.5$-fold for the 1 mM MAA data set.

Principal component analysis was used to extract characteristic patterns from the six microarray data sets. 5624 genes responding to either $1 \mathrm{mM}$ MAA or $5 \mathrm{mM}$ MAA were selected based on the combined criteria of |foldchange $\mid>2$ SD from mean and $p<0.005$ at one or more time points. The data were then pre-processed by logarithm 2 transformation of the expression ratio for each gene and by normalizing each gene's ratio to a mean value of zero and to a SD of 1 across the set of 6 arrays. Matrix $A$, which represents the gene expression data under all 6 microarray conditions, was decomposed by the singular value decomposition: $A=U V^{T}$, where both $U$ (an $N \times M$ matrix) and $V$ (an $M \times M$ matrix) are orthogonal matrices, and (an $M \times M$ matrix) is diagonal. The loading matrix $V$ consists of the weights of individual genes in the principal components. $\mathrm{V}^{T}$ is the transpose of $\mathrm{V}$. We denote $X=U$, where the principal component scores are specified.

\section{Motif enrichment analysis}

Flexmodule_motif, a de novo motif discovery function based on Gibbs motif sampler implemented in CisGenome [19], was used to identify DNA motifs overrepresented within $1 \mathrm{~kb}$ upstream of the transcription start sites of sets of genes either up or down regulated by MAA. The DNA motifs that best matched those overrepresented motifs were identified using STAMP, a database for alignment and similarity search for DNA motifs [20]. Next, Fisher's exact test was applied to test whether these known motifs are enriched in genes of each group compared to control gene sets. Two sets of genes were used as background controls: 1) genes expressed at a modest or high level in untreated TM3 cells (microarray signal intensity $>200$ ) that were not responsive to MAA treatment (|expression fold-change $\mid<1.2$ following $5 \mathrm{mM}$ MAA treatment at 3, 8 and $24 \mathrm{~h}$ ); and 2) genes expressed in TM3 cells at a low to undetectable level (microarray signal intensity $<50$ ) and $\mid$ expression fold-change $\mid<1.2$ at all three time points following MAA treatment. 
Ingenuity Pathway Analysis, GO enrichment and heat maps The list of MAA-regulated genes was analyzed using Ingenuity Pathway Analysis software (Ingenuity Systems, Mountain View, CA) based on gene ontology, biological processes, molecular function, and genetic networks. This software maps the biological relationship of uploaded genes into networks based on published literature for each gene. The biological function network, for example, identifies biological functions and diseases that are most significant to the data set. Genes that met the threshold criteria for MAA responsiveness and were associated with biological functions or diseases in the Ingenuity Pathway knowledge base were considered for further analysis. Fischer's exact test was used to calculate a $p$-value to determine the probability that each biological function/disease assigned to the data set is due to chance alone. A relevancy score was assigned to each network in the data set to estimate the relevancy of the network to the gene list uploaded. A higher relevancy score means that the network is more relevant to the gene list entered. Top pathways in each network are listed according to their $p$-values. Classified gene lists were analyzed for enrichment of Gene Ontology functional annotation clusters using DAVID, a web accessible bioinformatics database $[21,22]$. Heat maps were drawn using Java Treeview [23].

\section{Results}

\section{Overall impact of MAA on TM3 cell gene expression}

The impact of MAA treatment on mouse TM3 cell gene expression was determined in cells treated with either 1 mM or 5 mM MAA for 3,8 or $24 \mathrm{~h}$. These concentrations of MAA were chosen based on earlier reports demonstrating similar MAA concentrations in plasma of rodents exposed to toxic doses of ethylene glycol monomethyl ether [10] and in urine of humans exposed to ethylene glycol monomethyl ether in occupational situations [11]. Under these conditions, MAA did not cause any loss of TM3 cell viability over the course of at least 48 $\mathrm{hr}$. The three time points were selected to distinguish early MAA response genes (putative direct targets, whose expression does not require de novo protein synthesis) from late response genes (secondary response genes) [24]. RNA samples representing each time point were analyzed on expression microarrays in direct comparison to vehicle-treated controls.

Principal component analysis was applied to evaluate the expression patterns in the six microarrays. The first two of 6 principal components account for more than $70 \%$ of the variance in the overall data set (Fig. 1A). The pattern of the first component represents genes showing a steady increase in expression, or a steady decrease in expression, at both MAA concentrations (Fig. 1B), while the second component represents genes that show small

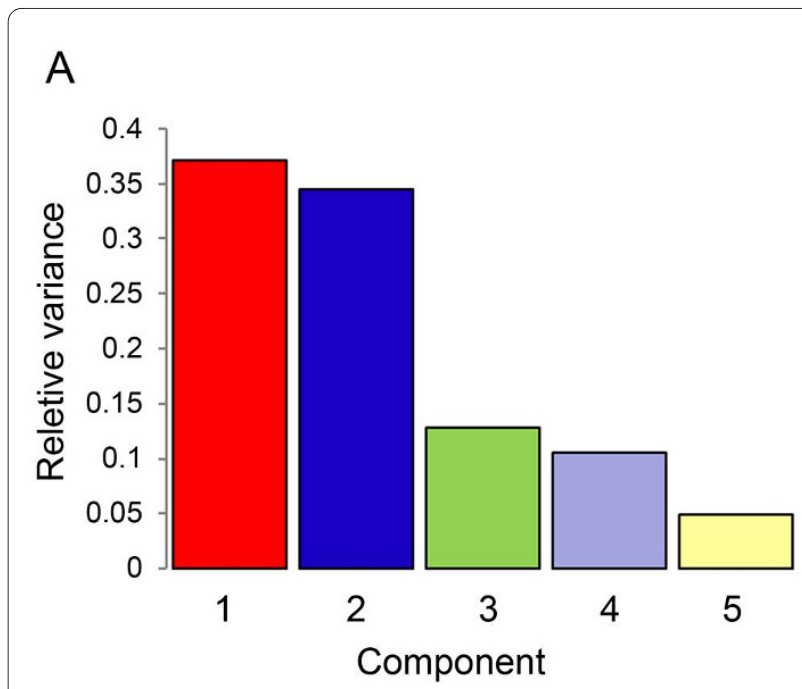

B
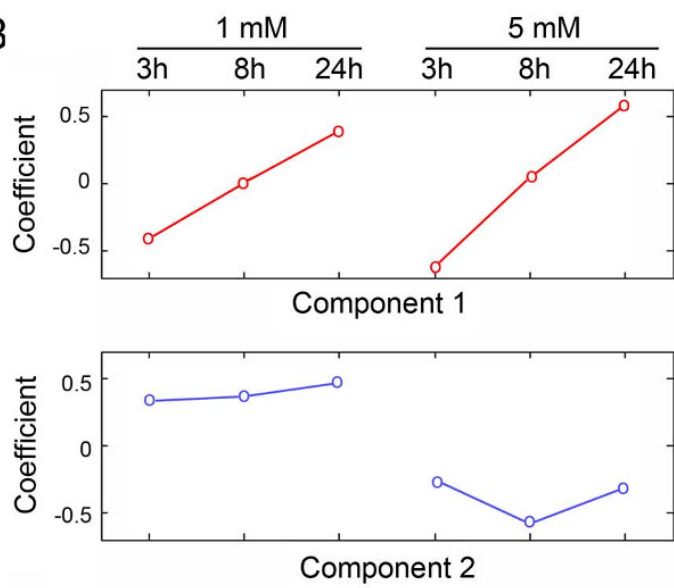

Figure 1 Principal component analysis of six microarray data sets. Plots of relative variance (A) and the coefficients of first and second components (B) are shown. Each coefficient indicates the weight of a particular treatment in the principal component. The first principal component represents a measure of expression change over time. The second coefficient captures information about the expression pattern affected by MAA dosage.

changes in expression as a function of time at $1 \mathrm{mM}$ MAA as compared to $5 \mathrm{mM}$ MAA (Fig. $1 \mathrm{~B}$ and Additional file 1). Based on these analyses, $37 \%$ of the variance in the data can be explained by the treatment duration, while $34 \%$ of the variance is due to different responses at low vs. high MAA concentrations. Most of the genes that show a different response at each MAA concentration show a more significant response to $5 \mathrm{mM}$ MAA than 1 mM MAA, as would be expected (Fig. 2 and Additional file 1). To explore the genes and pathways that may potentially contribute to MAA toxicity, we focused our analysis on the effect of $5 \mathrm{mM}$ MAA, and describe below common pathways triggered by both $1 \mathrm{mM}$ and $5 \mathrm{mM}$ MAA. 


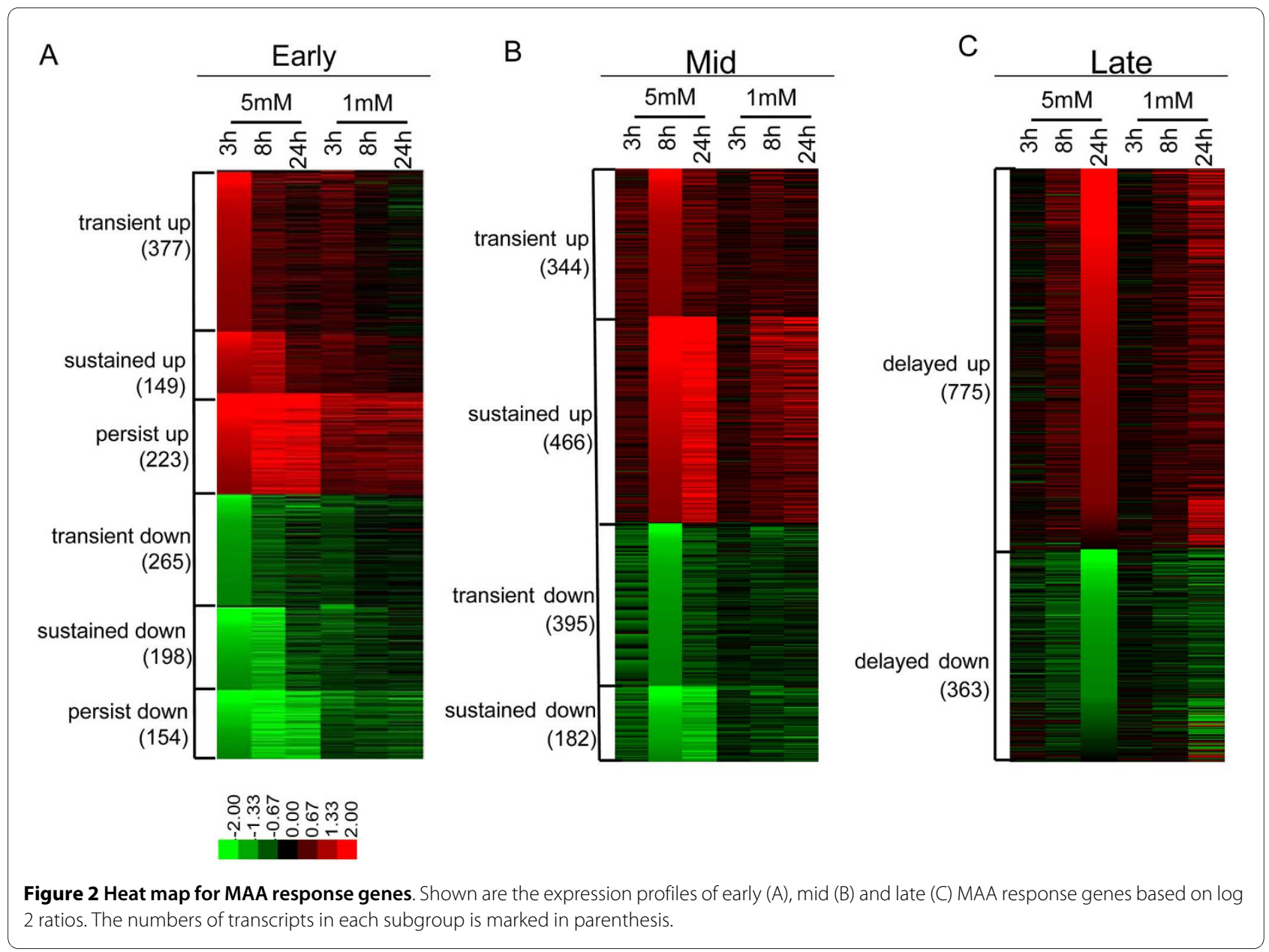

3,912 of the 41,174 microarray probes met the threshold criteria (absolute log 2 ratio $>2$ SD above the mean and $p<0.005)$, corresponding to an apparent false discovery rate (FDR) of $0.64 \%$, for at least one of the three $5 \mathrm{mM}$ MAA time points after elimination of redundant probes. The levels of 1,382, 2,122 and 2,226 transcripts were significantly altered by MAA at $3 \mathrm{~h}, 8 \mathrm{~h}$ and $24 \mathrm{~h}$, respectively. A complete list of the MAA-responsive genes together with their expression ratios and fluorescence signal intensities at each time point is provided in Additional file 2. Ingenuity Pathway Analysis was used to map the biological relationship of the MAA-responsive genes into networks constructed based on published literature about each gene (Table 1). Gene networks that responded to MAA include reproductive system development and function, embryonic development and tissue morphology. Disease categories that were impacted by MAA include reproductive system disease, inflammatory disease, inflammatory response, connective tissue disorders and skeletal and muscular disorders. The biological functions affected by MAA include cellular movement, cell- to-cell signaling and interaction, cellular development and cell death (Table 1).

\section{Clustering and functional analysis by significance and differential expression}

The impact of MAA treatment was further investigated by classification of the 3,912 MAA-responsive genes using a binary flagging system termed Total Flag Sum (TFS) [18], whereby each gene is categorized based upon its expression ratio and $p$-value in each of the three microarray experiments. Genes that responded to MAA rapidly, i.e., by the $3 \mathrm{~h}$ MAA time point, were distinguished from the mid-response genes (response by $8 \mathrm{~h}$ ) and the late responders (response by $24 \mathrm{~h}$ ) (Table 2 and Additional file 2). A total of 1,366 early response genes were identified, which were further categorized based on the persistence of their response to MAA (Table 2 and Fig. 2A). Among the early response genes, 642 genes responded at the $3 \mathrm{~h}$ time point but not at $8 \mathrm{~h}$ (early transient response genes), with 377 transcripts induced and 265 transcripts repressed. 106 of these early transient genes participate in transcriptional regulation, as deter- 
Table 1: Top biological functions and networks affected by MAA

\begin{tabular}{|c|c|c|}
\hline Name & $p$-value range & No. molecules \\
\hline \multicolumn{3}{|l|}{ A. Top Biological Functions: Diseases and Disorders } \\
\hline Reproductive System Disease & $3.45 \mathrm{E}-10-4.68 \mathrm{E}-04$ & 160 \\
\hline Inflammatory Disease & $1.76 \mathrm{E}-09-1.23 \mathrm{E}-03$ & 507 \\
\hline Inflammatory Response & $1.18 \mathrm{E}-08-1.77 \mathrm{E}-03$ & 279 \\
\hline Connective Tissue Disorders & $5.10 \mathrm{E}-07-1.23 \mathrm{E}-03$ & 44 \\
\hline Skeletal and Muscular Disorders & $5.10 \mathrm{E}-07-1.23 \mathrm{E}-03$ & 516 \\
\hline \multicolumn{3}{|l|}{ B. Molecular and Cellular Functions } \\
\hline Cellular Movement & 7.17E-12 - 1.81E-03 & 327 \\
\hline Cell-To-Cell Signaling and Interaction & $3.03 \mathrm{E}-11-1.53 \mathrm{E}-03$ & 333 \\
\hline Cellular Growth and Proliferation & $3.74 \mathrm{E}-10-1.75 \mathrm{E}-03$ & 492 \\
\hline Cellular Development & $7.76 \mathrm{E}-08-1.81 \mathrm{E}-03$ & 455 \\
\hline Cell Death & $8.78 \mathrm{E}-07-1.69 \mathrm{E}-03$ & 490 \\
\hline \multicolumn{3}{|c|}{ C. Physiological System Development and Function } \\
\hline Tissue Development & $3.47 \mathrm{E}-10-1.53 \mathrm{E}-03$ & 353 \\
\hline Cardiovascular System Development \& Function & $5.55 \mathrm{E}-08-1.56 \mathrm{E}-03$ & 169 \\
\hline Organismal Development & $5.55 \mathrm{E}-08-1.36 \mathrm{E}-03$ & 240 \\
\hline Hematological System Development \& Function & $7.98 \mathrm{E}-08-1.75 \mathrm{E}-03$ & 348 \\
\hline Immune Cell Trafficking & $1.58 \mathrm{E}-07-1.77 \mathrm{E}-03$ & 206 \\
\hline \multicolumn{3}{|c|}{ D. Associated Network Functions (top five gene network functions) } \\
\hline \multicolumn{3}{|c|}{ 1. Reproductive System Development and Function, Embryonic Development, Tissue Morphology. } \\
\hline \multicolumn{3}{|c|}{ 2. Infection Mechanism, Dermatological Diseases and Conditions, Organismal Injury and Abnormalities. } \\
\hline \multicolumn{3}{|c|}{ 3. Carbohydrate Metabolism, Small Molecule Biochemistry, Molecular Transport. } \\
\hline \multicolumn{3}{|c|}{ 4. Behavior, Nervous System Development and Function, Gene Expression. } \\
\hline \multicolumn{3}{|c|}{ 5. Cardiovascular System Development and Function, Embryonic Development, Tissue Development. } \\
\hline \multicolumn{3}{|c|}{$\begin{array}{l}\text { Shown is a summary of results of Ingenuity Pathway bio-functional analysis to identify biological functions or disease networks that are most } \\
\text { significant to the } 5 \text { mM MAA data set. Genes that met the threshold criteria defined in Materials and Methods and were associated with } \\
\text { biological functions or diseases in the Ingenuity Pathway Analysis Knowledge Base were considered in the analysis. Each network includes a } \\
\text { set of molecules, which usually perform more than one function. Fischer's exact test was used to calculate a } p \text {-value indicating the probability } \\
\text { that each biological function and/or disease assigned to the data set is due to chance alone. Shown is the } p \text {-value range, which indicates the } \\
\text { lowest and highest } p \text {-values corresponding to all molecules included in each network, and the total number of molecules present in the } \\
\text { network. }\end{array}$} \\
\hline
\end{tabular}

mined by gene functional annotation analysis using DAVID [21,22] (Table 3 and Additional file 3), and could contribute to the complex changes in the transcriptome associated with MAA treatment. Indeed, 102 of the 1,366 early response genes encode DNA-binding proteins, 60 of which showed a transient response to MAA (Additional file 4). Interestingly, 32 of these 102 early response DNAbinding proteins have been linked to developmental processes by DAVID analysis (Fig. 3). Moreover, 31 of the 102 genes contain zinc finger domains (Additional file 4). Functions have not been determined for many of these genes; however, two of the induced zinc-finger protein genes, Egr1 and Egr2, are notable insofar as Egr1-Egr4 double-mutant male mice are characterized by physiologically low serum testosterone levels and atrophy of andro- gen-dependent organs due to the loss of luteinizing hormone production in the pituitary gland [25]. Moreover, Egr1 is a key transcription factor implicated in the development and progression of prostate cancer [26].

Early response genes whose expression was altered by MAA at $3 \mathrm{~h}$ and $8 \mathrm{~h}$, but not at $24 \mathrm{~h}$, were designated early sustained up or early sustained down genes (Table 2 and Additional file 2; TFS groups 3.110 and 3.220, respectively). These genes are enriched in several cancer-related functional clusters, including insulin-like growth factor binding (Htra3, Igfbp5, Cyr61, Wisp1), GTP binding (Tubb6, Tgtp, Rnd1, Gbp1, Rab40b, Gbp2, Rhobtb1, Rabl3, Gbp3), cytokine activity (Clcf1, Kitl, Socs2, Cxcl5, Tnfsf11, Cxcl1, Cx3cl1, Il12a) and negative regulation of apoptosis (Cln8, Clcf1, Nuak2, Kitl, Dlx1, Notch1, Bcl2, 
Serpinb9, Clec2 d, Nr4a1, Fastkd2, Rassf5, Elmo1) (see Additional file 3 for complete listing of genes). Corresponding KEGG pathways include cytokine and receptor interaction, TLR-Jak-STAT signaling pathway, and phosphatidylinositol signaling. Other early genes whose response to MAA was maintained at $8 \mathrm{~h}$ and $24 \mathrm{~h}$ were designated early persistent genes (Table 3 and Additional file 2; TFS groups 3.111 and 3.222); examples include genes implicated in cytokine receptor interaction and calcium signaling (Additional files 3 and 5). 1,387 mid-response genes responded to MAA at $8 \mathrm{~h}$, while 1,138 late-response genes did not respond to MAA until $24 \mathrm{~h}$ (Figs. 2B, C and Table 2). These secondary response genes are enriched in a variety of signaling pathways and functional clusters, such as macromolecule metabolic process (Atf3, Adra1b, Fbxl16, Frk, Gls, Nanos1, Pcyt1b), cell adhesion (Arhgap6, Col1a2, Col6a2, Kitl, Tek, Thbs1, Tsc1), interferon-activated genes (Ifi202b, Ifi203, Ifi204, Ifi205), genes active in the extracellular space (Acp5, Csf1r, Muc2, Nppb, Ramp3), T cell activation and differentiation (Cd74, H2-DMa, Il15, Il7, Itgal) and metabolism of xenobiotics by cytochrome P450 and other enzymes (see Additional file 3 for complete listing of genes).

\section{DNA motifs associated with TM3 cell transcriptional responses}

CisGenome [19] was used to discover de novo motifs overrepresented in the sets of genes up or down regulated genes by MAA at each time point. GC rich motifs were found to be over represented in the proximal promoters of MAA up regulated genes. Motif comparison against the TRANSFAC database [27] revealed that these GC rich motifs are most similar to two known motifs, SP1 and KROX, which correspond to the binding sites for transcription factors of the SP1 and EGR families, respectively. Both motifs are enriched in MAA-regulated genes compared to a background gene set comprised of TM3 cell non-expressed genes, with enrichment scores ranging up to 2.1 (Additional file 6). However, motif enrichment was not observed when using as background a set of TM3 cell expressed genes that did not respond to MAA (Additional file 6). Thus, while SP1 and KROX/EGR motifs are overrepresented in some MAA-induced genes, these motifs also characterize TM3 cell expressed genes more generally.

Impact of MAA on genes associated with testicular function MAA had a wide range of effects on gene products important for testosterone biosynthesis, spermatogenesis, and testicular development and function (Fig. 4 and Additional file 7). For example, sex hormone binding globulin $(S h b g)$ was up regulated by $5 \mathrm{mM}$ MAA at all 3 time points, while the induction of Cyp11a1, Rhox5 (Pem) and $H o x b 13$ and the repression of Insl3 were seen at the $24 \mathrm{~h}$ MAA time point.

\section{Common response genes and pathways activated at low MAA concentration}

Early, mid and late MAA response genes were also identified in the $1 \mathrm{mM}$ MAA-treated TM3 cells. Early response genes common to the $1 \mathrm{mM}$ and $5 \mathrm{mM}$ MAA treatments (482 genes; Additional file 8) are expected to be enriched in direct targets of MAA; 294 of these genes were induced and 188 were repressed by MAA. DAVID analysis showed that phosphatidylinositol/phospholipase C/calcium signaling and cytokine/cytokine receptor interactions were the most significantly enriched pathways for the common early gene set. Pathways enriched in the common mid and late response genes include cell adhesion and focal adhesion (Additional file 9).

\section{Real time qPCR validation}

To confirm the results of the microarrays, qPCR analysis was performed for three genes that were induced (Rasgrp2, Itpka and Kcnab328; Fig. 5A) and for three genes that were repressed by MAA at all three time points (Pcdhb15, Abca9 and Ly96; Fig. 5B). Rasgrp2 and Itpka were of particular interest since our earlier studies had shown that MAA induces the Map kinase pathway and the PI3 kinase pathway in tsA201 cells [9]. Fig. 5 shows that the overall trends of expression seen by qPCR were consistent with the microarray data, although the fold-changes did not always match those of the microarray data, as is commonly seen.

\section{Discussion}

MAA is the active metabolite of the industrial chemical ethylene glycol monomethyl ether, a widely studied testicular toxicant. Presently, we characterize changes in gene expression induced by MAA in the cultured testicular Leydig cell model TM3. This investigation, carried out as a time course of MAA exposure, was designed to gain further insight into the range of changes in gene expression that MAA induces, including gene responses that could contribute to the testicular toxicity that is a hallmark of MAA exposure. The TM3 cell line was chosen based on our earlier finding that these cells are responsive to MAA, which induces changes in the expression of several genes related to androgen synthesis and activity [9]. MAA did not cause any changes in TM3 cell viability over the course of at least $48 \mathrm{hr}$; nevertheless, we observed extensive changes in TM3 cell gene expression. 3,912 genes were altered in their expression by $5 \mathrm{mM}$ MAA, the plasma MAA concentration associated with germ cell toxicity in mice [10]; 1,168 of these genes responded in 
Table 2: Classification of MAA responsive genes by duration of MAA treatment required to elicit response

\begin{tabular}{|c|c|c|c|c|c|}
\hline Group & Sub-group & 3 h MAA & 8 h MAA & 24 h MAA & Genes in each subgroup (number) \\
\hline \multirow[t]{8}{*}{ Early (1366 genes) } & early transient up & Up & - & - & 351 \\
\hline & early transient up & Up & - & Up & 26 \\
\hline & early sustained up & Up & Up & - & 149 \\
\hline & early persistent up & Up & Up & Up & 223 \\
\hline & early transient down & Down & - & - & 245 \\
\hline & early transient down & Down & - & Down & 20 \\
\hline & early sustained down & Down & Down & - & 198 \\
\hline & early persistent down & Down & Down & Down & 154 \\
\hline \multirow[t]{4}{*}{ Mid (1387 genes) } & mid transient up & - & Up & - & 344 \\
\hline & mid sustained up & - & Up & Up & 466 \\
\hline & mid transient down & - & Down & - & 395 \\
\hline & mid sustained down & - & Down & Down & 182 \\
\hline \multirow[t]{2}{*}{ Late (1138 genes) } & late up & - & - & Up & 775 \\
\hline & late down & - & - & Down & 363 \\
\hline
\end{tabular}

The $5 \mathrm{mM}$ MAA-responsive genes were divided into 3 groups, early response $(3 \mathrm{~h})$, mid response $(8 \mathrm{~h})$ and late response ( $24 \mathrm{~h})$ based on the minimum period of MAA exposure that was required to evoke a gene response compared to control cells. The direction of response is indicated as Up (up-regulation), Down (down-regulation) or - (no significant response). 21 other genes that did not meet these MAA response criteria are shown in Additional file 2.

common to $1 \mathrm{mM}$ MAA, which is more relevant to the exposure level seen in humans [11]. As discussed below, the gene expression changes and associated cellular pathways impacted by MAA in this cellular model parallel some of the toxicities associated with MAA exposure, suggesting that these findings in TM3 cells may serve as a model for MAA-induced toxicities in other cell types as well.

\section{Rapid MAA induction of zinc finger transcription factors}

Analysis of the time dependence of MAA-induced gene responses allowed us to identify genes that respond to MAA rapidly (within $3 \mathrm{~h}$ ), as well as genes whose altered in expression persists and could potentially serve as biomarkers of MAA exposure. Environmental chemicalinduced toxicities are often associated with early gene responses [28]; therefore, genes that respond to MAA rapidly may provide insights into the pathophysiological changes caused by MAA. Presently, we found that 102 of the 1,366 early response genes encode DNA-binding proteins, 32 of which have been linked to developmental processes (Fig. 3). Interestingly, 60 of the early response DNA-binding proteins showed a transient response to MAA, i.e., gene induction responses seen at $3 \mathrm{~h}$ were largely reversed by $8 \mathrm{~h}$ (Additional file 4), suggesting a feedback loop for the regulation of transcriptional activity by MAA. For example, ATF1 (activating transcription factor 1), a bZIP domain containing protein belonging to the CREB family, was down regulated 2-fold after $3 \mathrm{~h}$ of
MAA treatment, while CREB5 (cAMP response elementbinding 5), another CREB family transcription factor, was up regulated 3.7-fold. Upon activation, CREB binds as a dimer to the cAMP response element (CRE), where it promotes the recruitment of the transcriptional coactivators CREB binding protein (CBP) and p300 [29]. The impact of this opposite regulation of these two CREB family proteins by MAA on the expression of CRE-regulated genes is unknown. One CRE-regulated gene, Egr1, was induced 4.5-fold after $3 \mathrm{~h}$ MAA exposure. EGR1 is a zinc-finger transcription factor that binds to a GC-rich motif, as do several other zinc finger proteins, which may activate or repress their targets by acting in combination [30]. Previously, evolutionarily conserved zinc finger transcription factor binding sites recognized by WT1, EGR1, SP1, SP2, AP2 and GATA1 were identified in the promoters of 24 differentially expressed prostate cancer genes from eight mammalian species [26], suggesting these zinc finger proteins play a pivotal role in the prostate. Here, both EGR1 and SP1 binding sites were found to be overrepresented in TM3 Leydig cell-expressed genes compared to TM3 cell non-expressed genes. Further studies, including gene knock-down and in vivo transcription factor binding assays, are needed to clarify whether these putative sites are true EGR1/SP1 binding sites, whether they are functional, and what impact their binding may have on TM3 cell gene activation or repression. 
Table 3: Gene functional clusters affected by MAA for each sub-group

\begin{tabular}{|c|c|c|c|c|}
\hline Response & Top term & Enrich-ment score & Cluster gene count & $p$-value \\
\hline \multirow[t]{3}{*}{ Early transient } & GO:0045449, Regulation of transcription & 5.96 & 106 & 3.23E-07 \\
\hline & GO:0044424, Intracellular part & 5.8 & 211 & $1.11 \mathrm{E}-06$ \\
\hline & GO:0065007, Biological regulation & 5.54 & 124 & $6.15 \mathrm{E}-07$ \\
\hline \multirow[t]{3}{*}{ Early sustained } & GO:0050794, Regulation of cellular process & 3.41 & 66 & $1.85 \mathrm{E}-04$ \\
\hline & IPR000867: Insulin-like growth factor-binding protein, IGFBP & 3.04 & 4 & $6.28 \mathrm{E}-04$ \\
\hline & Domain: von Willebrand factor, type $C$ & 2.91 & 4 & 4.81E-04 \\
\hline \multirow[t]{3}{*}{ Early persistent } & GO:0048731, System development & 2.19 & 48 & $1.19 \mathrm{E}-03$ \\
\hline & GO:0048514, Blood vessel morphogenesis & 2.13 & 9 & 2.17E-03 \\
\hline & GO:0044421, Extracellular region part & 2.07 & 43 & $6.46 \mathrm{E}-03$ \\
\hline \multirow[t]{3}{*}{ Mid transient } & GO:0007155, Cell adhesion & 3.11 & 34 & $3.28 \mathrm{E}-04$ \\
\hline & GO:0044237, Cellular metabolic process & 2.25 & 347 & $1.60 \mathrm{E}-04$ \\
\hline & IPR004021: HIN-200/IF120x & 2.13 & 5 & $9.10 \mathrm{E}-04$ \\
\hline \multirow[t]{3}{*}{ Mid sustained } & GO:0044421, Extracellular region part & 8.22 & 109 & 7.30E-10 \\
\hline & IPR015493: Protocadherin beta & 6.56 & 15 & $1.68 \mathrm{E}-12$ \\
\hline & IPR003128: Villin headpiece & 4.39 & 5 & $2.85 \mathrm{E}-05$ \\
\hline \multirow[t]{3}{*}{ Late } & GO:0044421, Extracellular region part & 10.34 & 165 & $5.60 \mathrm{E}-12$ \\
\hline & GO:0048856, Anatomical structure development & 4.4 & 144 & $1.10 \mathrm{E}-05$ \\
\hline & GO:0044449, Contractile fiber part & 3.47 & 12 & $1.75 \mathrm{E}-04$ \\
\hline
\end{tabular}

Listed are the top three gene functional clusters affected by MAA as determined using DAVID database. The top term for each cluster, the cluster enrichment score and the number of regulated genes in each cluster are listed.

\section{Impact of MAA on protein kinase pathways}

Protein kinases play a significant role in MAA-induced toxicity, and protein kinase inhibitors can minimize these effects [31]. Of the 3,912 MAA-regulated genes identified in our study, 91 code for protein kinases, as determined by Ingenuity Pathway Analysis (Additional file 11). These kinases are associated with amino acid metabolism, posttranslational modification, small molecule biochemistry, cell death and cell movement. The top canonical pathways affected include inositol metabolism, axonal guidance signaling, FAK signaling, germ cell-Sertoli cell junction signaling, and nicotinate and nicotinamide metabolism. MAA can act as a hormone sensitizer to enhance the transcriptional activity of several nuclear receptors without itself being a hormone mimetic, although the underlying mechanism is only partly understood [9,32]. Our previous studies demonstrated that the potentiation of androgen receptor activity by MAA requires tyrosine kinase signaling that is independent of the RAS-MEK-ERK signaling pathway but requires PI3 kinase activity [9]. Consistently, in this study, we observed that the phosphoinositide/phospholipase $\mathrm{C} / \mathrm{cal}$ cium pathway is enriched in the set of early response genes in common to both $1 \mathrm{mM}$ and $5 \mathrm{mM}$ MAA treatment. Several key factors in this pathway are induced by MAA, including phospholipases C, PI3 kinases, and IP3 receptor (Additional file 9). Both the ERK and PI3 kinase pathways have been suggested to be responsible for Egr1 up regulation [33,34]. Further studies will be required to identify the underlying molecular mechanism whereby MAA activates these signaling pathways leading to induction of Egr1 and its downstream targets.

\section{Effect of MAA treatment on histone genes}

Several genes encoding histone proteins were induced by MAA treatment. Thus, Hist1h1d, Hist1h4k, and Hist $2 h 2 a a 1$ were early MAA response genes, Hist $1 h 1 c$, Hist $2 h 2 b e$, and Hist $3 h 2 b a$ were mid-response genes, and 9 other Hist genes, including 6 encoding histone 1 proteins, were late response genes (Additional file 2). H1 histones stabilize compact, higher order structures of 
chromatin, regulate gene expression, and participate in chromatin-based processes like DNA replication and repair [35]. The altered expression of this linker histone in MAA-treated cells could destabilize chromatin architecture and contribute to malignant transformation or genetic disorders. Hist1h1d is significantly up regulated in rat testis within $4 \mathrm{~h}$ of MAA treatment, and this change has been correlated with increased acetylation of core histones [36]. The induction of mouse Hist1h1d seen in our study is consistent with that earlier report, and supports the utility of the mouse TM3 model for identifying gene changes that may be linked to MAA toxicity. It will be interesting to determine the relationship between histone up regulation and the other gene expression changes caused by MAA.

\section{Biological pathways affected by MAA}

Many of the prominent toxicological effects of MAA or the parent toxicant, ethylene glycol monomethyl ether, are well characterized [37] and several studies have elucidated effects of MAA on intracellular signaling pathways and changes in gene expression [3,36]. Using Ingenuity Pathway Analysis we identified pathways related to repro-

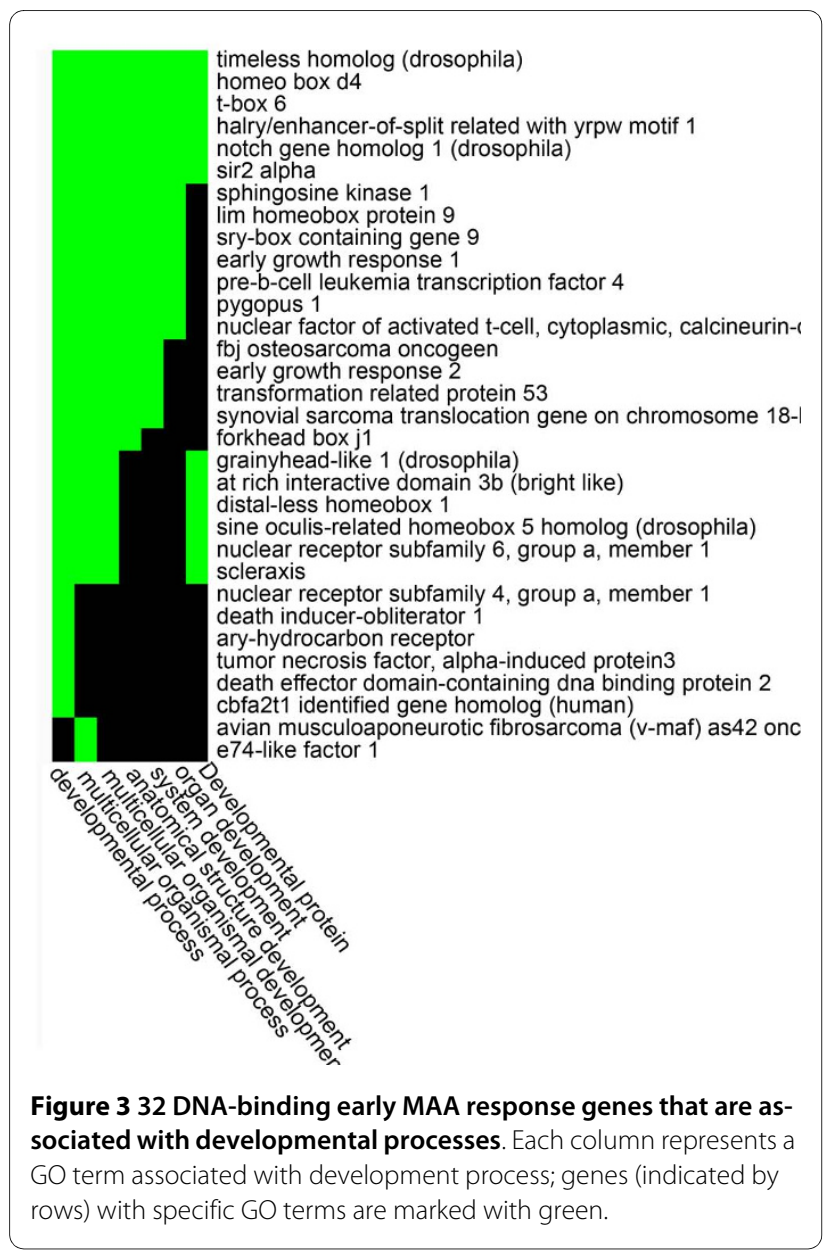

ductive system development and function, embryonic development and tissue morphology to be the most affected by MAA. Moreover, at all three time points examined, MAA affected genes associated with several disease pathways, reproductive system disease, inflammatory disease, connective tissue disorders and skeletal and muscular disorders. Alteration of these pathways in the corresponding target tissues could contribute to some of the physiological diseases and disorders that have been associated with MAA exposure, including MAA-induced disruption of neurogenesis and limb and digit differentiation in mice [1], hematopoiesis [38], cell death [39] and immune system disorders [40-42]. MAA also induced significant changes in the expression of membrane proteins, which is reflected in the dysregulation of genes involved in cell-to-cell signaling, cell adhesion and cell mobility as indicated by Ingenuity Pathway functional analysis (Table 1 ). Indeed, a majority of the genes that were induced or repressed by MAA under all treatment conditions belong to this category (Table 3 and Additional file 3). Moreover, 32 of 102 early MAA response genes encoding DNA-

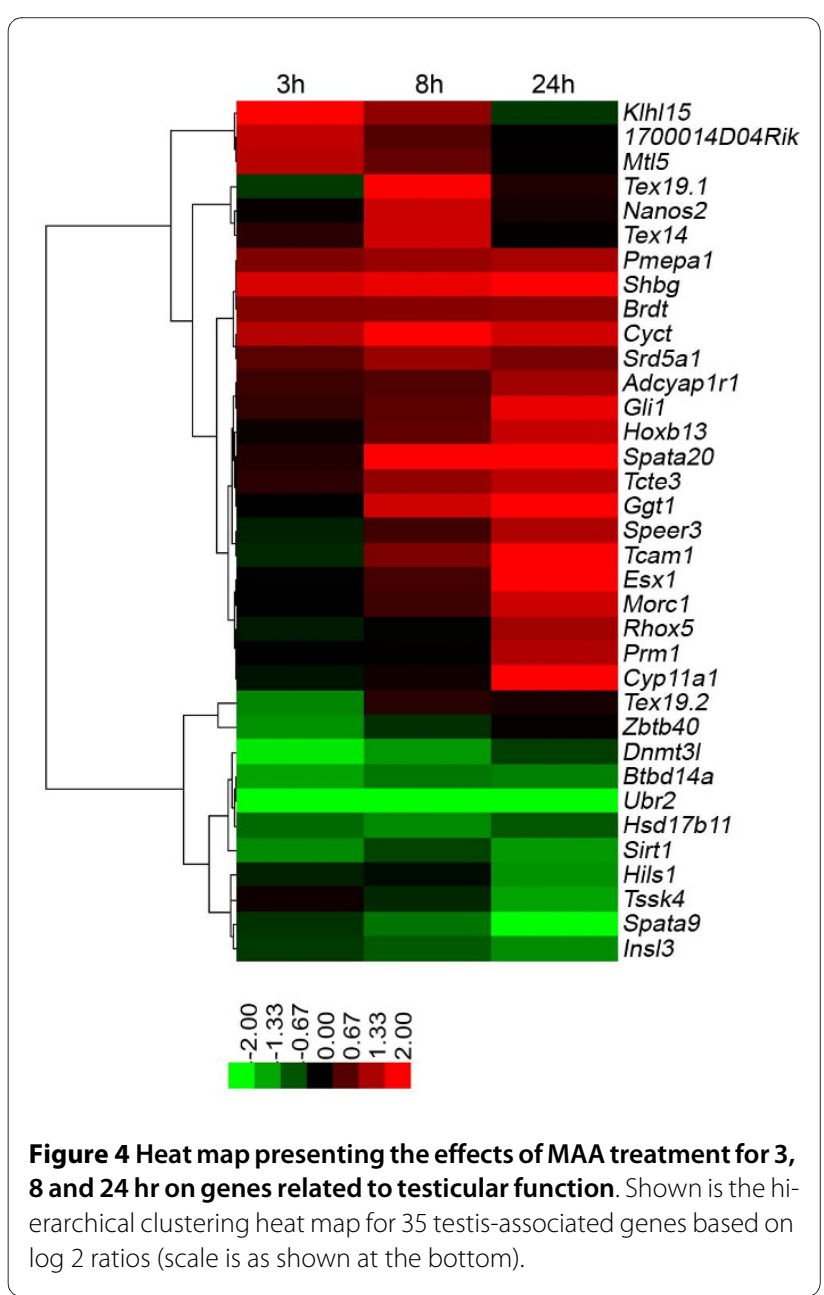




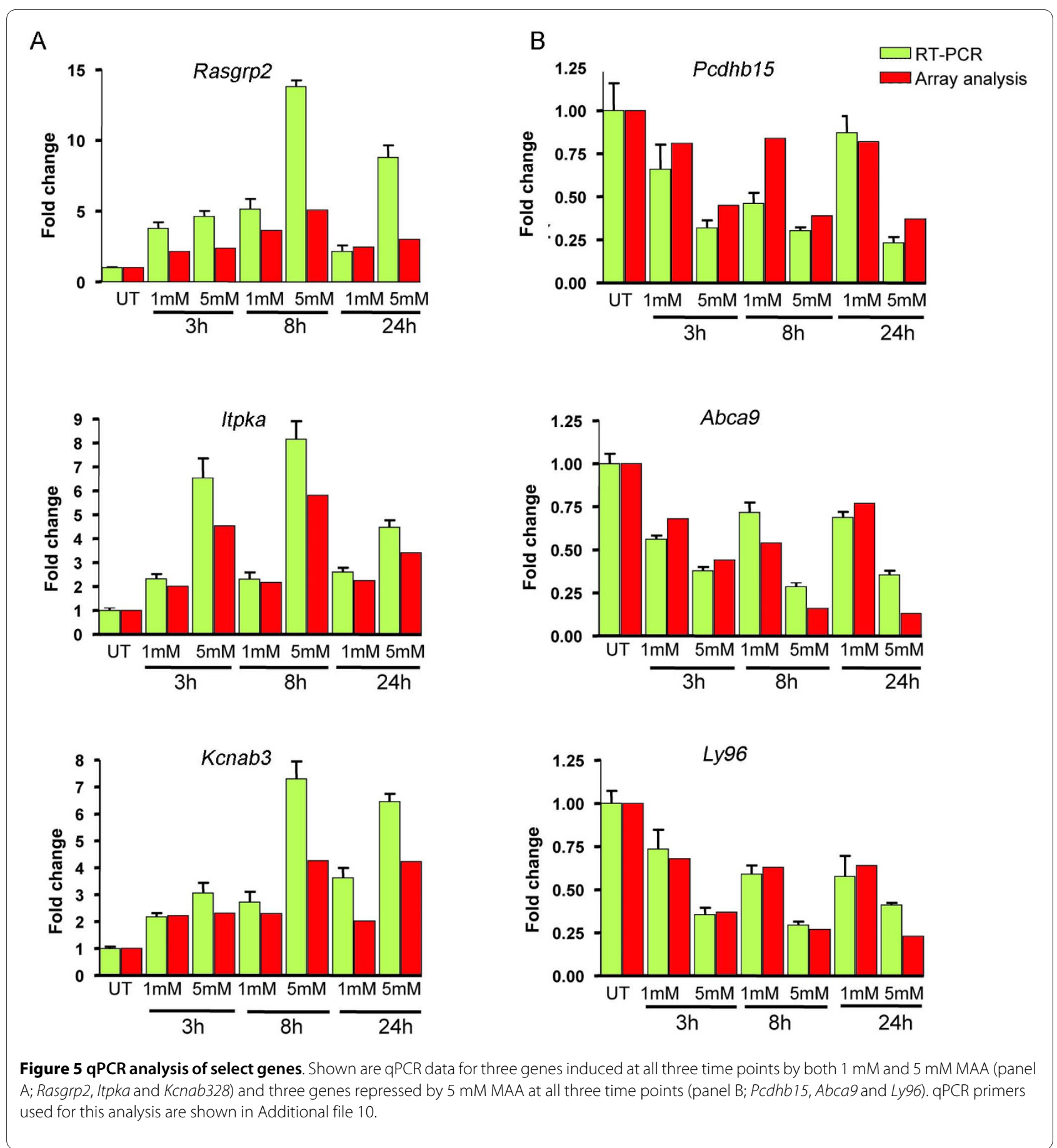

binding proteins are associated with developmental processes (Fig. 3).

Genes related to testicular function whose expression was significantly altered by MAA include Cyp11a1, Hsd17b11, Shbg and Insl3 and Rhox5 (Fig. 4 and Additional file 7), which encode proteins involved in testosterone biosynthesis or development of the male reproductive system. Changes in the expression of these genes might impact testosterone biosynthesis/availability and influence spermatocyte survival. CYP11A1 cleaves the side chain of cholesterol to yield pregnenolone, the initial step in the pathway leading from cholesterol to steroid hormone production [43]. HSD17B11 is a hydroxysteroid dehydrogenase that is required for synthesis of androstenedione, a precursor of testosterone [44]. Insulin-like 3 (INSL3; Leydig insulin-like peptide) is important in testis descent [45], with male mice mutant for Insl3 exhibiting cryptorchidism or defects in testis 
descent due to abnormal gubernaculum development [46]. Steroid hormone binding globulin (SHBG) binds androgens and its over-expression can cause testosterone depletion and toxicity [47,48]. The expression of Cyp17a1, which catalyzes the 17-hydroxylase and lyase activities required for testosterone synthesis [49], was increased in MAA-treated cells, as determined by qPCR, although this change was not seen in the microarray analysis. Further studies are required to determine if these changes affect Leydig cell testosterone production, which could have a direct impact on germ cell survival and toxicity. MAA also induced two homeobox genes, Rhox5 and Hoxb13, which have been implicated in the modulation of androgen receptor-regulated gene expression in Sertoli cells and in prostate, respectively $[50,51]$. Rhox5, which is considered a Sertoli cell marker gene but is also expressed at a low level in Leydig cells in vivo [52], is thus an MAAresponsive transcription factor that may mediate some of the effects of MAA on downstream targets [50]. Finally, MAA down regulated estrogen receptor- $\alpha$, suggesting the MAA can also impact estrogenic signaling in testicular cells. Of note, MAA also disrupts estrogenic signaling in MCF7 cells in vitro and in the mouse uterus in vivo [53].

Metabolic labeling studies establish that MAA can be activated to the thioester 2-methoxyacetyl- coenzyme A, which enters the tri-carboxylic acid cycle [1]. Conceivably, just as acetyl coenzyme A is funneled into multiple metabolic pathways, 2-methoxyacetyl-coenzyme A may enter multiple pathways, including tri-carboxylic acid cycle, fatty acid metabolism and amino acid metabolism with affects on cellular metabolism and gene expression. Acetyl coenzyme A is also essential for histone acetylation, a key event in gene transcription [54], suggesting that 2-methoxyacetyl-coenzyme A could also affect that process. We observed significant MAA-induced changes in expression of genes involved in cellular metabolism, oxidation status, transcription and gene expression, as might be caused by a metabolite which can enter and impact several key cellular metabolic and regulatory pathways. Further studies using inhibitors and metabolites of specific pathways are needed to test these hypotheses.

\section{Conclusions}

In this study, we monitored the progressive changes in gene expression induced by MAA in a cultured Leydig cell model and detected extensive changes in TM3 cell gene expression. The MAA-responsive pathways identified are linked to reproductive system development and function, embryonic development and tissue morphology. These MAA-induced perturbations of cellular and biological functions may help elucidate the testicular pathophysiological responses induced by MAA exposure and identify useful biomarkers of MAA toxicity.

\section{Additional material}

Additional file 1 Principal component analysis of microarray data
sets.
Additional file 2 Expression ratio and TFS of 5 mM MAA response
transcripts and transcripts of each subgroup.
Additional file 3 Gene functional clusters affected by 5 mM MAA for
each sub-group as determined using DAVID database, together with
the KEGG pathways enriched in each group.
Additional file 4 Expression ratio and TFS of 102 early response genes
identified as DNA-binding proteins by their GO terms.
Additional file 5 KEGG Pathways associated with 5 mM MAA early
response genes. In each pathway, the MAA-responsive genes are marked
by a red asterisk.
Additional file 6 Enriched motif analysis of MAA response genes.
Additional file 7 Impact of MAA on testis expressed genes.
Additional file 8 Expression ratio and TFS of response transcripts
common to both 1 mM and 5 mM MAA treatment in each sub-group.
Additional file 9 Gene functional clusters and KEGG pathways
enriched in each group of 1 mM and 5 mM MAA common response
genes.
Additional file 10 Mouse qPCR primer sets and Genebank accession
numbers.
Additional file 115 mM MAA regulated kinases as identified by Inge-
nuity Pathway Analysis (IPA).

Competing interests

The authors declare that they have no competing interests.

Authors' contributions

GB and DJW conceived and designed the experiments, GB performed the experiments, YZ, GB and DJW analyzed the data and wrote the paper, and DJW managed the overall design and execution of the project. All authors read and approved the final manuscript.

\section{Acknowledgements}

Supported in part by National Institutes of Health grant 5 P42 ES07381, Superfund Research Program at Boston University (to D.J.W.). The authors thank Dr. Alan Dombkowski, Wayne State University, for processing the microarray data sets using Rosetta Resolver.

\section{Author Details}

Division of Cell and Molecular Biology, Department of Biology, Boston University, Boston, MA 02215, USA

Received: 25 April 2010 Accepted: 18 June 2010

Published: 18 June 2010

\section{References}

1. Welsch F: The mechanism of ethylene glycol ether reproductive and developmental toxicity and evidence for adverse effects in humans. Toxicol Lett 2005, 156:13-28.

2. Li LH, Wine RN, Chapin RE: 2-Methoxyacetic acid (MAA)-induced spermatocyte apoptosis in human and rat testes: an in vitro comparison. J Androl 1996, 17:538-549.

3. Bagchi G, Waxman DJ: Toxicity of ethylene glycol monomethyl ether: impact on testicular gene expression. Int J Andro/ 2008, 31:269-274.

4. De Gendt K, Swinnen JV, Saunders PT, Schoonjans L, Dewerchin M, Devos A, Tan K, Atanassova N, Claessens F, Lecureuil C, Heyns W, Carmeliet P, Guillou F, Sharpe RM, Verhoeven G: A Sertoli cell-selective knockout of the androgen receptor causes spermatogenic arrest in meiosis. Proc Natl Acad Sci USA 2004, 101:1327-1332

5. Xu Q, Lin HY, Yeh SD, Yu IC, Wang RS, Chen YT, Zhang C, Altuwaijri S, Chen LM, Chuang KH, Chiang HS, Yeh S, Chang C: Infertility with defective spermatogenesis and steroidogenesis in male mice lacking androgen receptor in Leydig cells. Endocrine 2007, 32:96-106.

6. Tirado OM, Martinez ED, Rodriguez OC, Danielsen M, Selva DM, Reventos J, Munell F, Suarez-Quian CA: Methoxyacetic acid disregulation of 
androgen receptor and androgen-binding protein expression in adult rat testis. Biol Reprod 2003, 68:1437-1446.

7. Takamiya M, Lambard S, Huhtaniemi IT: Effect of bisphenol A on human chorionic gonadotrophin-stimulated gene expression of cultured mouse Leydig tumour cells. Reprod Toxicol 2007, 24:265-275.

8. Mather JP: Establishment and characterization of two distinct mouse testicular epithelial cell lines. Biol Reprod 1980, 23:243-252.

9. Bagchi $G$, Hurst $C H$, Waxman DJ: Interactions of methoxyacetic acid with androgen receptor. Toxicol Appl Pharmacol 2009, 238:101-110.

10. Terry KK, Elswick BA, Stedman DB, Welsch F: Developmental phase alters dosimetry-teratogenicity relationship for 2-methoxyethanol in CD-1 mice. Teratology 1994, 49:218-227.

11. Shih TS, Liou SH, Chen CY, Smith TJ: Urinary 2-methoxy acetic acid accumulation in response to 2-methoxy ethanol exposure. Arch Environ Health 2001, 56:20-25.

12. Holloway MG, Laz EV, Waxman DJ: Codependence of growth hormoneresponsive, sexually dimorphic hepatic gene expression on signal transducer and activator of transcription $5 \mathrm{~b}$ and hepatic nuclear factor 4alpha. Mol Endocrinol 2006, 20:647-660

13. Schmittgen TD, Livak KJ: Analyzing real-time PCR data by the comparative CT method. Nat Protocols 2008, 3:1101-1108.

14. Chen R, Li L, Butte AJ: AILUN: reannotating gene expression data automatically. Nat Methods 2007, 4:879

15. Wauthier V, Sugathan A, Meyer RD, Dombkowski AA, Waxman DJ: Intrinsic Sex Differences in the Early Growth Hormone Responsiveness of Sex-Specific Genes in Mouse Liver. Mol Endocrinol 2010, 24:667-678

16. Holloway MG, Miles GD, Dombkowski AA, Waxman DJ: Liver-specific hepatocyte nuclear factor-4alpha deficiency: greater impact on gene expression in male than in female mouse liver. Mol Endocrinol 2008, 22:1274-1286

17. Weng L, Dai H, Zhan Y, He Y, Stepaniants SB, Bassett DE: Rosetta error model for gene expression analysis. Bioinformatics 2006, 22:1111-1121

18. Clodfelter KH, Holloway MG, Hodor P, Park SH, Ray WJ, Waxman DJ: Sexdependent liver gene expression is extensive and largely dependent upon signal transducer and activator of transcription $5 b$ (STAT5b): STAT5b-dependent activation of male genes and repression of female genes revealed by microarray analysis. Mol Endocrinol 2006 , 20:1333-1351.

19. Ji H, Jiang H, Ma W, Johnson DS, Myers RM, Wong WH: An integrated software system for analyzing ChIP-chip and ChIP-seq data. Nat Biotech 2008, 26:1293-1300.

20. Mahony S, Benos PV: STAMP: a web tool for exploring DNA-binding motif similarities. Nucl Acids Res 2007:gkm272.

21. Dennis G Jr, Sherman BT, Hosack DA, Yang J, Gao W, Lane HC, Lempicki RA: DAVID: Database for Annotation, Visualization, and Integrated Discovery. Genome Biol 2003, 4:P3.

22. Huang da W, Sherman BT, Lempicki RA: Systematic and integrative analysis of large gene lists using DAVID bioinformatics resources. Nat Protoc 2009, 4:44-57.

23. Saldanha AJ: Java Treeview--extensible visualization of microarray data. Bioinformatics 2004, 20:3246-3248

24. Zhou Q, Shima JE, Nie R, Friel PJ, Griswold MD: Androgen-regulated transcripts in the neonatal mouse testis as determined through microarray analysis. Biol Reprod 2005, 72:1010-1019.

25. Tourtellotte WG, Nagarajan R, Bartke A, Milbrandt J: Functional compensation by Egr4 in Egr1-dependent luteinizing hormone regulation and Leydig cell steroidogenesis. Mol Cell Biol 2000 , 20:5261-5268

26. Eisermann K, Tandon S, Bazarov A, Brett A, Fraizer G, Piontkivska H: Evolutionary conservation of zinc finger transcription factor binding sites in promoters of genes co-expressed with WT1 in prostate cancer. BMC Genomics 2008, 9:337.

27. Matys V, Kel-Margoulis OV, Fricke E, Liebich I, Land S, Barre-Dirrie A, Reuter I, Chekmenev D, Krull M, Hornischer K, Voss N, Stegmaier P, LewickiPotapov B, Saxel H, Kel AE, Wingender E: TRANSFAC and its module TRANSCompel: transcriptional gene regulation in eukaryotes. Nucleic Acids Res 2006, 34:D108-110.

28. Irwin RD, Boorman GA, Cunningham ML, Heinloth AN, Malarkey DE, Paules RS: Application of toxicogenomics to toxicology: basic concepts in the analysis of microarray data. Toxicol Pathol 2004, 32(Suppl 1): $72-83$.
29. Carlezon JWA, Duman RS, Nestler EJ: The many faces of CREB. Trends Neurosci 2005, 28:436-445.

30. Philipsen S, Suske G: A tale of three fingers: the family of mammalian Sp/XKLF transcription factors. Nucleic Acids Res 1999, 27:2991-3000.

31. Jindo T, Wine RN, Li LH, Chapin RE: Protein kinase activity is central to rat germ cell apoptosis induced by methoxyacetic acid. Toxicol Pathol 2001, 29:607-616.

32. Jansen MS, Nagel SC, Miranda PJ, Lobenhofer EK, Afshari CA, McDonnell DP: Short-chain fatty acids enhance nuclear receptor activity through mitogen-activated protein kinase activation and histone deacetylase inhibition. Proc Natl Acad Sci USA 2004, 101:7199-7204

33. Cabodi S, Morello V, Masi A, Cicchi R, Broggio C, Distefano P, Brunelli E, Silengo L, Pavone F, Arcangeli A, Turco E, Tarone G, Moro L, Defilippi P: Convergence of integrins and EGF receptor signaling via PI3K/Akt/ FoxO pathway in early gene Egr-1 expression. J Cell Physiol 2009, 218:294-303.

34. Silva PN, Soares JA, Brasil BS, Nogueira SV, Andrade AA, de Magalhaes JC, Bonjardim MB, Ferreira PC, Kroon EG, Bruna-Romero O, Bonjardim CA: Differential role played by the MEK/ERK/EGR-1 pathway in orthopoxviruses vaccinia and cowpox biology. Biochem J 2006 398:83-95.

35. Happel N, Doenecke D: Histone $\mathrm{H} 1$ and its isoforms: contribution to chromatin structure and function. Gene 2009, 431:1-12.

36. Wade MG, Kawata A, Williams A, Yauk C: Methoxyacetic acid-induced spermatocyte death is associated with histone hyperacetylation in rats. Biol Reprod 2008, 78:822-831.

37. Welsch F, Blumenthal GM, Conolly RB: Physiologically based pharmacokinetic models applicable to organogenesis: extrapolation between species and potential use in prenatal toxicity risk assessments. Toxicol Lett 1995, 82-83:539-547.

38. Holladay SD, Comment CE, Kwon J, Luster MI: Fetal hematopoietic alterations after maternal exposure to ethylene glycol monomethyl ether: prolymphoid cell targeting. Toxicol Appl Pharmacol 1994, 129:53-60.

39. Nagano K: Testicular atrophy of mice induced by ethylene glycol mono alkyl ethers (author's transl). Sangyo Igaku 1979, 21:29-35.

40. Exon JH, Mather GG, Bussiere JL, Olson DP, Talcott PA: Effects of subchronic exposure of rats to 2-methoxyethanol or 2-butoxyethanol: thymic atrophy and immunotoxicity. Fundam App/ Toxicol 1991, 16:830-840

41. Kawamoto T, Matsuno K, Kayama F, Hirai M, Arashidani K, Yoshikawa M, Kodama Y: Acute oral toxicity of ethylene glycol monomethyl ether and diethylene glycol monomethyl ether. Bull Environ Contam Toxicol 1990, 44:602-608.

42. Smialowicz RJ, Riddle MM, Williams WC: Methoxyacetaldehyde, an intermediate metabolite of 2-methoxyethanol, is immunosuppressive in the rat. Fundam App/ Toxicol 1993, 21:1-7.

43. Guo IC, Hu MC, Chung BC: Transcriptional regulation of CYP11A1. J Biomed Sci 2003, 10:593-598.

44. Chai Z, Brereton P, Suzuki T, Sasano H, Obeyesekere V, Escher G, Saffery R, Fuller $\mathrm{P}$, Enriquez C, Krozowski Z: 17 beta-hydroxysteroid dehydrogenase type XI localizes to human steroidogenic cells. Endocrinology 2003, 144:2084-2091.

45. Lague E, Tremblay JJ: Estradiol represses insulin-like 3 expression and promoter activity in MA-10 Leydig cells. Toxicology 2009, 258:101-105.

46. Kumagai J, Hsu SY, Matsumi H, Roh JS, Fu P, Wade JD, Bathgate RA, Hsueh AJ: INSL3/Leydig insulin-like peptide activates the LGR8 receptor important in testis descent. J Biol Chem 2002, 277:31283-31286.

47. Jeyaraj DA, Grossman G, Petrusz P: Altered bioavailability of testosterone in androgen-binding protein-transgenic mice. Steroids 2005, 70:704-714

48. Johanson G: Toxicity review of ethylene glycol monomethyl ether and its acetate ester. Crit Rev Toxicol 2000, 30:307-345.

49. Payne $\mathrm{AH}$, Youngblood $\mathrm{GL}$ : Regulation of expression of steroidogenic enzymes in Leydig cells. Biol Reprod 1995, 52:217-225.

50. Hu Z, Dandekar D, O'Shaughnessy PJ, De Gendt K, Verhoeven G, Wilkinson MF: Androgen-Induced Rhox Homeobox Genes Modulate the Expression of AR-Regulated Genes. Mol Endocrinol 2010, 24:60-75

51. Norris JD, Chang C-Y, Wittmann BM, Kunder RS, Cui H, Fan D, Joseph JD, McDonnell DP: The Homeodomain Protein HOXB13 Regulates the Cellular Response to Androgens. Mol Cell 2009, 36:405-416. 
52. Lindsey JS, Wilkinson MF: Pem:A Testosterone- and LH-Regulated Homeobox Gene Expressed in Mouse Sertoli Cells and Epididymis. Dev Biol 1996, 179:471-484.

53. Henley DV, Mueller S, Korach KS: The short-chain fatty acid methoxyacetic acid disrupts endogenous estrogen receptor-alphamediated signaling. Environ Health Perspect 2009, 117:1702-1706,

54. Takahashi H, McCaffery JM, Irizarry RA, Boeke JD: Nucleocytosolic acetylcoenzyme a synthetase is required for histone acetylation and global transcription. Mol Cell 2006, 23:207-217.

doi: 10.1186/1477-7827-8-65

Cite this article as: Bagchi et al., Impact of methoxyacetic acid on mouse Leydig cell gene expression Reproductive Biology and Endocrinology 2010, 8:65

Submit your next manuscript to BioMed Central and take full advantage of:

- Convenient online submission

- Thorough peer review

- No space constraints or color figure charges

- Immediate publication on acceptance

- Inclusion in PubMed, CAS, Scopus and Google Scholar

- Research which is freely available for redistribution

Submit your manuscript at www.biomedcentral.com/submit
C) Biomed Central 